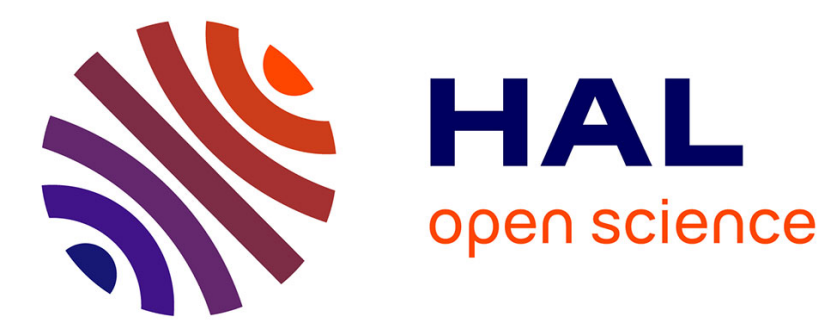

\title{
Clinically oriented real-time monitoring of the individual's risk for deep tissue injury.
} Sigal Portnoy, Nicolas Vuillerme, Yohan Payan, Amit Gefen

\section{To cite this version:}

Sigal Portnoy, Nicolas Vuillerme, Yohan Payan, Amit Gefen. Clinically oriented real-time monitoring of the individual's risk for deep tissue injury.. Med Biol Eng Comput, 2011, 49 (4), pp.473-83. 10.1007/s11517-011-0754-y . hal-00591952

\section{HAL Id: hal-00591952 https://hal.science/hal-00591952}

Submitted on 11 May 2011

HAL is a multi-disciplinary open access archive for the deposit and dissemination of scientific research documents, whether they are published or not. The documents may come from teaching and research institutions in France or abroad, or from public or private research centers.
L'archive ouverte pluridisciplinaire HAL, est destinée au dépôt et à la diffusion de documents scientifiques de niveau recherche, publiés ou non, émanant des établissements d'enseignement et de recherche français ou étrangers, des laboratoires publics ou privés. 


\title{
Clinically-Oriented Real-Time Monitoring of the Individual's Risk for Deep Tissue Injury
}

\author{
Sigal Portnoy ${ }^{1}$, Nicolas Vuillerme ${ }^{2,3}$, Yohan Payan ${ }^{2}$, Amit Gefen ${ }^{1}$ \\ ${ }^{1}$ Department of Biomedical Engineering, Tel Aviv University, Israel \\ ${ }^{2}$ Laboratoire TIMC-IMAG, Faculté de Médecine, CNRS-Universite Joseph Fourier, Grenoble \\ France
}

${ }^{3}$ CIC-IT 805, INSERM/AP-HP, Hôpital Raymond Poincaré, EA 4497, Garches, France

Number of words: 5278

Number of words in the abstract: 200

Number of figures : 6

Number of tables : 2

Corresponding Author:

Prof. Amit Gefen

Department of Biomedical Engineering

Faculty of Engineering

Tel Aviv University

Tel Aviv 69978

$\underline{\text { Israel }}$

Tel: $+972-3-640-8093$

Fax: +972-3-640-5845

E-mail: gefen@eng.tau.ac.il

T appear in Medical \& Biological Engineering \& Computing 


\begin{abstract}
Spinal cord injury patients are under daily risk for developing deep tissue injury a severe pressure ulcer that initiates in soft tissues at the bones' proximity. We aimed to formulate a patient-specific biomechanical model that can continuously monitor internal tissue stresses in real-time. We adopted a formulation solving an axisymmetric contact problem of a finite-thickness, elastic layer (soft tissue) and a rigid spherical indentor (ischial tuberosity). We utilized finite element analyses to expand the formulation for large deformations. Sensitivity analyses showed that the soft tissue mechanical properties are the most influential factors in this modeling. We then used synthetic surface pressure data and actual surface pressures recorded under the buttocks of 5 paraplegic wheelchair-users to demonstrate clinical feasibility. Output parameters were designed to be simple so that they can be easily interpreted by the user. Specifically, we calculated peak and average internal von Mises stress and stress dose, under each buttock, and also a time-dependent stress asymmetry index, to account for frequency of posture adjustments. Inter-subject variability was higher than the intra-subject variability. The heaviest subject had the highest maximal and average peak internal soft tissue stress. We believe that this method holds a high potential for clinical applications.
\end{abstract}

Keywords: Pressure ulcer; Rehabilitation; Sitting; Wheelchair; Biomechanical model. 


\section{INTRODUCTION}

Spinal cord injury (SCI) patients are under daily risk for developing deep tissue injury (DTI), which is a severe pressure ulcer that typically initiates in soft tissues at the proximity of the ischial tuberosity (IT) bones $[2,4,8,15,16,20]$. DTI has recently become a consensus pathway of injury, which is reflected in the International Pressure Ulcer Guidelines published jointly by the European and American Pressure Ulcer Advisory Panels (see www.epuap.org and www.npuap.org, respectively). While surface pressure measurements are believed to be effective in alerting wheelchairusers against focal pressures that may cause skin injury [18], these measurements cannot predict dangerous internal tissue loading [1,12]. For example, a similar pressure map may register under the buttocks of a heavy person with sharp ITs and a thin person with blunt ITs; however, their susceptibility to DTI depends on the IT curvature as well as the thickness of the soft tissues, as demonstrated by MRI-based finite element (FE) analyses conducted by Elsner and Gefen [7] and Sopher et al. [21]. Patient-specific FE analyses demand relatively high computer power, at least for large tissue deformations, which is the case for the buttocks area, and the model creation process cannot be easily automated [3]. Furthermore, monitoring sitting pressures through pressure mats is mostly reserved to rehabilitation institutes due to high costs. Therefore, internal loads are presently unmonitored - neither in the clinical setting nor in the patient's daily surroundings.

Previous studies [1,11] showed that real-time patient-specific monitoring of internal soft tissue strains and stresses under the ITs is, in principle, feasible. However, the Agam and Gefen [1] model did not directly account for the individual's soft tissue thickness under the ITs. The Linder-Ganz et al. [11] model was more anatomically accurate but required the computer power of a laptop to run. Here we sought to develop a biomechanical model that is simple enough for running on low computer power, i.e. can be programmed on low-cost chips, in real-time, but that still holds essential individual anatomical characteristics. We further aimed to establish "user-friendly" output parameters that can be easily interpreted by users. The biomechanical model is presented herein and its clinical feasibility is demonstrated. The present study applies to SCI patients, who are considered more prone to DTI with respect to other patient populations $[2,4,8,15,16,20]$. Note that we do not aim at demonstrating prevention capabilities of the method at this stage, but rather, we 
present the algorithm and provide a proof-of-concept. Demonstrating prevention capacities will require further clinical studies that are underway.

\section{METHODS}

\subsection{Biomechanical Model}

In order to evaluate internal stresses in sub-dermal soft tissues at the proximity of the IT in real-time, using low computing power and low hardware costs, we adopted a formulation solving an axisymmetric contact problem of a finite-thickness, transversely isotropic elastic layer and a rigid spherical indentor [14]. The IT bone edge is modeled as a hemisphere with radius $R$ (Fig. 1a). Due to the bodyweight, the IT hemisphere is indenting, to a depth of $z_{0}$, against a soft tissue layer that effectively represents the mechanical contributions of the overlying muscle, fat and skin. A contact radius $a$ is formed between the IT and soft tissue layer. The downward displacement of the IT is given by $z_{0}=w+R-\sqrt{R^{2}-a^{2}}$ (Fig. 1a). Assuming small deformations of the soft tissue at this stage of the modeling, $w \approx a^{2} / 2 R$ [14]. The thickness of the soft tissue is $h$. The load applied to the IT by the bodyweight at time $t$ is $P(t)$, which induces an equal and measurable reaction force at the surface of the body (Fig. 1a). The contact between the IT and the soft tissue can be considered as either free-slip, i.e. the bone can slide freely on the overlying soft tissue or as no-slip, meaning that there is no movement between the bone and the soft tissue (see further below).

The formulation of the stress tensor in the soft tissue for the IT-soft tissue contact problem depicted in Fig. 1a is as follows. For a no-slip IT-soft tissue interface [14]:

$$
\left.\begin{array}{c}
\sigma_{r r}=-c_{13} \frac{a^{2}-r^{2}}{2 R h} \\
\sigma_{z z}=-c_{33} \frac{a^{2}-r^{2}}{2 R h} \\
\sigma_{r z}=-\frac{c_{44} r}{R h}(h-z)
\end{array}\right\} \forall r \in[0, a] \quad \text { where } \quad a=\left(\frac{4 R h P(t)}{\pi c_{33}}\right)^{\frac{1}{4}}
$$

where $C_{i j}$ are the constitutive parameters of the soft tissue (given later in eq. 3). 
For free-slip IT-soft tissue interface, the stress tensor is [14]:

$$
\left.\begin{array}{rl}
\sigma_{r r} & =-\frac{\left(c_{11}-c_{12}\right) c_{13}}{8 c_{11} R h}\left(2 a^{2}-r^{2}\right) \\
\sigma_{r z} & =0 \\
\sigma_{z z} & =-\frac{c_{11} c_{33}-c_{13}^{2}}{2 c_{11} R h}\left(a^{2}-r^{2}\right)
\end{array}\right\} \forall r \in[0, a] \text { where } a=\left[\frac{4 P(t) c_{11} R h}{\pi\left(c_{11} c_{33}-c_{13}^{2}\right)}\right]^{\frac{1}{4}}
$$

Assuming now an isotropic soft tissue layer, the constitutive parameters of the soft tissue are:

$$
\begin{aligned}
& c_{11}=c_{33}=\frac{(1-v) \alpha E}{(1+v)(1-2 v)} \\
& c_{12}=c_{13}=\frac{v \alpha E}{(1+v)(1-2 v)} \\
& c_{44}=\frac{\alpha E}{2(1+v)}
\end{aligned}
$$

where $E$ and $v$ are the effective elastic modulus and Poisson's ratio of the soft tissue, respectively. A geometry function $\alpha(R / h)$ was introduced herein to expand the formulation for determining soft tissue stresses under large deformations as well. The $\alpha(R / h)$ functions were characterized separately for the no-slip and free-slip IT-soft tissue contact conditions, using large-deformation FE models (described later on) of the geometry depicted in Fig. 1a.

The von Mises stresses in the soft tissue around the IT at each time point $t$ are calculated by:

$$
\sigma_{V M}(t, r, z)=\sqrt{\frac{\left[\sigma_{r r}-\sigma_{z z}\right]^{2}+{\sigma_{z z}}^{2}+\sigma_{r r}{ }^{2}+6 \sigma_{r z}{ }^{2}}{2}}
$$

In the real-world situation, slipping of the IT upon the soft tissue is hindered by interlacing fibers between the IT and the surrounding soft tissue, and possibly by some degree of tissue adhesion [19]. Since the real-world contact between the IT and soft tissue is neither frictionless nor completely bonded, we aimed at calculating the von Mises stress in the soft tissue assuming frictional IT-soft tissue contact. For this purpose, we used a weight-function with $\beta$ proportional to the IT-soft tissue friction coefficient, as follows: 


$$
\sigma_{V M}(t, r, z)=\beta\left(\sigma_{V M}^{n o-s l i p}(t, r, z)\right)+(1-\beta)\left(\sigma_{V M}^{\text {free-slip }}(t, r, z)\right)
$$

Substituting $\beta=1$ provides the no-slip solution and substituting $\beta=0$ provides the freeslip solution to the soft tissue stresses. An alternative approach was also explored, in which the weighted average of the two stress tensors (eq. 1 and eq. 2) was first calculated, followed by the calculation of the von Mises stress, according to eq. 4 . Differences between this approach and the algorithm expressed by eq. 5 did not exceed $7.5 \%$ in internal stress values, hence we proceeded with the eq. (5) option, consistent with the separate free-slip and no-slip FE analyses conducted to determine $\alpha(R / h)$.

Focusing on spatial peaks of soft tissue stresses, which appear at the IT-soft tissue contact [12], we limit eqs. (1) and (2) to the symmetry axis ( $r=0$; Fig. 1a) where soft tissue deformations maximize, and do not depend on the $z$ coordinate. The geometry functions $\alpha_{(R / h)}^{\text {noslip }}$ and $\alpha_{(R / h)}^{\text {freeslip }}$ mentioned above were determined by comparing von Mises stresses computed using eq. (5) for different $R / h$ values, with peak von Mises stresses computed with large-deformation FE simulations of corresponding geometries (Fig. 1). Specifically, each axisymmetric FE model (ABAQUS v. 6.9, SIMULIA, RI, USA) consisted of a rigid IT with radius $R$, and a soft tissue layer with thickness $h$, effective elastic modulus $E=25.3 \mathrm{kPa}$ (as measured for transversely indented flaccid muscle tissue by Palevski and colleagues) [17] and Poisson's ratio $v=0.49$ [10]. The soft tissue layer was modeled with 8-node biquadratic axisymmetric quadrilateral elements ("CGAX8R" in ABAQUS) with higher mesh density at the contact region (Fig. 1). We selected the mesh densities after gradually condensing the meshes until the resulted stresses did not change by more than $2 \%$. We analyzed internal soft tissue stresses where $R / h$ ranged between 0.5 to 2.3 ( $R$ was set to $20 \mathrm{~mm}$ and $h$ values ranged between $8.7 \mathrm{~mm}$ to $40 \mathrm{~mm}$ ), which represents a broad range of anatomical and pathoanatomical conditions characteristic to paraplegia, as previously measured by means of MRI [12]. We collected data from a total of $6 \mathrm{FE}$ model geometries (see $R / h$ values in Fig. 1d,e), applied with downward displacement so that the ratios of IT indentation depth over the undeformed soft tissue thickness were $20 \%$ to $50 \%$ at $6 \%$-steps. Each model was simulated with either no-slip or free-slip IT-soft tissue contact (Fig. 1). From the aforementioned FE simulations we obtained that $\alpha(R / h)$ behaves as a power law function (Fig. 1d,e): 


$$
\alpha=A\left(\frac{R}{h}\right)^{B}
$$

where the constants $A$ are 0.9718 and 0.0686 for the free-slip and no-slip contact conditions, respectively, and the constants $B$ are -0.277 and -0.285 for the free-slip and no-slip contact conditions, respectively (Fig. 1). This function was chosen since it provided better fit than polynomial functions and had just two parameters. Note that for the anatomically-relevant $R / h$ range, and in a large deformation regime, the $\alpha(R / h)$ functions mostly act to lower $\sigma_{V M}(t)$. The criterion for determining the $A$ and $B$ constants was a minimal root mean square error (RMSE) between the von Mises stresses predicted from eq. (5) and the peak von Mises stresses computed by FE simulations with corresponding $R / h$ geometry:

$$
R M S E=\sqrt{\frac{1}{N} \sum\left(\frac{\sigma_{V M}-\sigma_{V M, F E}}{\sigma_{V M, F E}}\right)^{2}}
$$

After introducing the $\alpha(R / h)$ functions in eq. (3), the mean RMSE were $5.4 \%$ for the no-slip and 3\% for the free-slip IT-soft tissue contact conditions, respectively, for the entire range of $R / h$. The maximal RMSE were $7.2 \%$ (at $R / h=0.8$ ) for the no-slip and $5.5 \%$ (at $R / h=2.3$ ) for the free-slip IT-soft tissue contact conditions, respectively. The correction factor has been calculated for $R / h$ in the undeformed configuration, so that it decreases with a decrease in the applied load (i.e. when loads are small the correction factor tends to 1). Nevertheless, real-world conditions involve IT indentation depths over undeformed soft tissue thicknesses in the range of 20-50\% (as applied herein) [12], and hence, in practice, all calculations involved large loads/deformations, and $R / h$ values that were higher than zero (at least 0.5 ).

In order to evaluate the history of soft tissue exposure to stresses over time, an internal stress dose (ISD) parameter was computed - as the numerical time integral of the peak internal von Mises stress in the soft tissue. At the occurrence of a load-relief, the ISD is reduced proportionally to the duration of the relief. Specifically, the ISD is multiplied by a factor of $1 / t$ (time in seconds), so that if a person lifts his buttock for $1 \mathrm{~s}$, there is no reduction in accumulated ISD, if he or she lifts it for $2 \mathrm{~s}$, the stress dose reduces to half and so on. 


\subsection{Sensitivity Analyses}

Subsequently, our analytical model was used to perform sensitivity analyses of intrinsic patient factors, i.e. anatomy and tissue mechanical properties. The elastic modulus of the soft tissue varied between $13.7 \mathrm{kPa}$ and $40 \mathrm{kPa}$ according to experimental data from loading flaccid muscle tissue perpendicularly to its fibers [17]. Poisson's ratios were varied between 0.49 and 0.499 , as soft tissues of the buttocks contain $\sim 80 \%$ water [10]. The geometry of the IT radii $(12 \mathrm{~mm}-25 \mathrm{~mm})$ and soft tissue thicknesses $(8 \mathrm{~mm}-40 \mathrm{~mm})$ were adopted from the MRI studies of Linder-Ganz and colleagues [12]. The parameter $\beta$, which is proportional to the IT-soft tissue friction coefficient, was varied through its entire range (0 to 1 ; eq. 5$)$. The results of the sensitivity analyses, presented in Table S1 (included with the supplementary material), revealed that the mechanical properties of the soft tissue $(E, v)$ are the most influential factors in this modeling, followed by the internal anatomy $(R, h)$. Accordingly, the impact of variation in soft tissue mechanical properties on the peak internal von Mises stress and ISD was tested via simulation of static and dynamic sitting conditions (see Section 2.3 below).

\subsection{Static and Dynamic Synthetic Data}

We simulated two possible wheelchair sitting conditions: static sitting and a dynamic loading during wheelchair propulsion. To simulate static sitting we created synthetic data consisting of 2 minutes of bilateral weight bearing, followed by load shifting to the monitored side for two minutes, then 5 seconds lift-off (zero load on the monitored buttock) and back to 2 minutes of bilateral weight bearing (Fig. 2). Simulations of dynamic sitting were created according to force patterns measured under the buttocks of wheelchair users during wheelchair propulsion [5] (Fig. 3). In each simulation we varied either the elastic modulus $E$ or the Poisson's ratio $v$ for the ranges defined in Section 2.2. In each simulation we calculated the internal peak von Mises stress and the ISD as function of time (Figs. 2,3).

\subsection{Determining the Optimal Pressure Sensing Area under the Ischial Tuberosity}

At this stage of the formulation, all parameters of our model are well-defined except the area on the pressure mat to be used for calculating body loads transferred through the IT on each side, $P(t)$. We therefore compared previously-published 
subject-specific MRI-based FE buttocks stress results [12] to our present stress calculations, made for different surface area sizes and for the same subjects, in order to test which surface pressure area produces stress values that best fit the MRI-FE calculations by Linder-Ganz and colleagues [12]. Specifically, we compared values of peak soft tissue von Mises stresses calculated by our present algorithm against patient-specific FE internal soft tissue von Mises stresses published by Linder-Ganz et al. [12] for the same healthy $(n=6)$ and paraplegic $(n=5)$ subjects during static sitting. For our calculations we assumed that the surface load under each IT, $P(t)$, was dispersed across a square of the buttock-sit contact area around the projection of the IT in the pressure map. The $P(t)$ force was hence calculated from pressure mat measurements acquired from each subject ("Tactilus", SensorProduct Co., NJ, USA), by multiplying the measured pressure [12] and different sizes of the aforementioned square. We assumed that $E^{\text {muscle }}=25.3 \mathrm{kPa}$ [17], $E^{f a t}=0.852 \mathrm{kPa}$ [9], $v=0.49$ [10] and $\beta=0.2$ [19] for all patients. The effective elastic modulus assigned to the soft tissue bulk of each individual subject was weighed using their individual MRI-measured [12] muscle-to-fat ratios (averaged for the left and right body sides; Table S2). A $5 \times 5 \mathrm{~cm}^{2}$ area size provided the best outcome in terms of an X-Y plot (Fig. 4a) and an average-difference (Bland-Altman) plot (Fig. 4b), and it is also physiologically reasonable - as a projection of the IT with a sufficiently large soft tissue region under it. Stresses calculated using the present model were compared with effective values of von Mises stress in muscle and fat tissues in Linder-Ganz et al. [12], which were correspondingly weighed together according to the subject-specific muscle-to-fat ratios. The average-difference plot comparing the present soft tissue stress data with the previous subject-specific MRI-based FE stress analyses of the same 11 subjects published by Linder-Ganz et al. [12], as shown in Fig. 4b, overall demonstrates good agreement between the two methods, as all data points in the plot are within the \pm 2 standard deviation (SD) boundaries of the difference (Fig. 4b), and the absolute mean difference between the methods is $\sim 4 \mathrm{kPa}$.

\subsection{Patient Studies}

The present algorithm was tested with sitting pressure data recorded from paraplegic patients in order to demonstrate its clinical feasibility. Specifically, pressure measurements between the wheelchair seat and the buttocks of five paraplegic subjects were previously recorded at a frequency of $1 \mathrm{~Hz}$ with a 256- 
sensors pressure mat ("Tactilus") by Linder-Ganz et al. [12]. An ethical committee approval was obtained for these studies and all patients gave their informed consent. We have selected a convenience sample, which reflects the midrange of patient body weights; the body characteristics and relevant personal information of the subjects are provided in Table S2. For the purpose of assessing feasibility of clinical application, we assumed, at this stage, that $E^{\text {muscle }}=25.3 \mathrm{kPa}[17], E^{f a t}=0.852 \mathrm{kPa}$ [9], $v=0.49$ [10] and $\beta=0.2$ [19] for all patients. The elastic modulus assigned to the soft tissue bulk of each subject was weighted using their individual MRI-measured [12] muscle-to-fat ratios (Table S2). Our algorithm for calculation of internal peak von Mises stresses was employed separately for the left and right buttock of each subject over pressure data recorded for a duration of 2 minutes of static sitting. A code was written (LabView 8.0, National Instruments Co., TX, USA) which finds the peak sitting pressure from one sensor (sensing area of $2.5 \times 2.5 \mathrm{~cm}^{2}$; “"Tactilus") under the left or right buttock in order to identify the location of the projection of the IT at each side. The code then chooses 4 sensors at this location that together form a square of $5 \times 5 \mathrm{~cm}^{2}$ which includes the peak pressure, and which also have the highest sum of pressure measurements. This code was verified by visually inspecting its selections of IT projection locations against the measured pressure maps. We computed the maximal internal peak von Mises stress in the soft tissue at each body side for each patient, as well as the averages of von Mises stresses over time. We then calculated the coefficient of variation (COV) for both intra-subject and inter-subject stress data.

A time-dependent soft tissue stress asymmetry index (SAI) was further defined, based on an analogue sitting pressure asymmetry index introduced in Linder-Ganz et al. [11]:

$$
S A I(t)=\log \left(\frac{\sigma_{V M}^{\text {left IT }}}{\sigma_{V M}^{\text {right IT }}}\right)
$$

This SAI is a measure of the extent of asymmetry in internal soft tissue load sharing between the two body sides. The SAI produces zero for ideally balanced sitting, is positive when the peak internal soft tissue stress is higher under the left IT and negative when the peak stress is higher under the right IT. We calculated the peak and average SAI per each patient and the COV for both intra-subject and inter-subject 
data. We further calculated the time integral of the SAI and the median SAI frequency as a measure of how often subjects change posture.

\section{RESULTS}

The synthetic data simulations (Figs. 2,3) demonstrated that consistent with Table $\mathrm{S} 1$, variations in $v$ overall had mild influence on the evaluated soft tissue stress and ISD, provided that $v$ was kept within the range of $0.49-0.496$, which well covers the physiologically-relevant range of Poisson's ratios for soft tissues [10]. Hence $E$ is the more important patient-specific mechanical property that needs to be considered in clinical application of the present method.

The maximal peak and average internal von Mises stresses calculated separately under the left and right IT of 5 paraplegic subjects (whose characteristics are provided in Table S2) are presented in Table 3. An example of the loads, peak internal von Mises stress and ISD under the left and right IT of subject D3 (Table S2) is plotted in Fig. 5. In a two-minutes period of static sitting, the soft tissues under the left IT of this particular subject consistently bore higher stresses and ISD than the soft tissues under his right IT, despite that at some periods, e.g. during the timeframe between 40s and $65 \mathrm{~s}$, the $P(t)$ at the left and right sides were approximately equal (Fig. 5). This indicates the importance of weighing the effect of the internal anatomy as well, which indeed demonstrates a slightly sharper IT and a thinner soft tissue layer at the left buttock side of this subject (Table S2), which caused the higher internal stresses at his left buttock side.

The averages of maximal peak internal von Mises stresses over time, as well as corresponding COV are calculated for both intra-subject and inter-subject data (Table 1). The heaviest subject, W5 (Table S2), had the highest internal von Mises stresses under his left IT. High values of COV of soft tissue stress were calculated for subject K4, who had the thickest soft tissue bulk (Table S2). The inter-subject variability, as determined by $53.4 \% \mathrm{COV}$ of the average of peak von Mises stresses across subjects, was higher than the intra-subject variability, for which the COV maximal value was $37.9 \%$ and which averaged below 12\% (Table 1). 
The SAI parameter (eq. 8) is plotted in Fig. 6 for all the paraplegic subjects (Table S2). Peak and average of peak internal SAI, COV of the SAI and median SAI frequency are presented in Table 2. Average SAI and corresponding COV are calculated for both intra-subject and inter-subject data (Table 2). The peak, average and time integral of SAI values calculated for subject K4 were at least 2-times higher than SAI values of the other subjects (Table 2, Fig. 6). Consistent with that, the median SAI frequency calculated for subject K4 was the maximal among the five subjects, indicating that subject K4 often shifted her weight from side to side.

\section{DISCUSSION}

In this study, we aimed at designing a patient-specific modeling method which is clinically-oriented and that will monitor in real-time and alert wheelchair users against high deep tissue stresses that may cause DTI. We employed clinically-oriented intuitive measures of the risk for DTI in an individual patient, being the current peak internal tissue stress and the stress history (ISD), as well as the balance between stresses in the right and left buttock sides (SAI) and its frequency of change and history. This entire set of parameters is based on the von Mises stress, which was selected because it is a scalar that weighs the contributions of the different components of the stress tensor (compression, tension and shear) together. In view of the target user communities - clinicians and perhaps the patients themselves - this is practical and efficient in terms of data reduction. The SAI in particular is a dimensionless number that indicates how often patients shift weight between sides. Weight shifting may temporarily relieve high loads under one IT, yet cause extreme loading on the contralateral IT. Asymmetry is a very important parameter used by clinicians to evaluate postures and load-bearing activities. Physicians, physical therapists and care-takers are accustomed to terminology concerning with asymmetry and hence it is a simple measure that provides data which clinicians can easily interpret. Quantification of internal stresses, as well as the weight shifting frequency, can provide clinicians with simple quantitative data concerning the patient's sitting habits (posture, load-asymmetry and load shifting frequency). Following continuous monitoring with the proposed system, patients can learn to regulate their internal soft tissue loading and adapt to safer sitting habits in term of DTI prevention. In other words, the system is useful for clinical evaluation as well as biofeedback applications. 
It should be noted that the concept of the SAI is considerably different than simply tracking the regulation of sitting pressures between the two body sides, because an inherent anatomical left-right asymmetry (demonstrated in most of the $R, h$ pairs of parameters in Table S2) imposes a need to apply uneven sitting pressures between the two buttock sides, in order to compensate for the anatomical asymmetry.

Previous models of our group, designed to predict patient-specific internal soft tissue stresses under the IT in real-time, were limited by several assumptions, i.e. modeling the soft tissue bulk as an infinite half-space (assumed in Herz's theory in Agam and Gefen [1]) or utilizing linear FE with no-slip between all tissues and requiring laptop computing power [13]. The modeling designed herein better approximates the actual anatomy of the buttocks by considering finite soft tissue thickness and frictional IT-soft tissue interaction, but still we were able to keep a closed-form analytical solution to the stress state, which is essential for real-time, low-computer power applications. In fact, the relative simplicity of the present modeling allows it to be programmed in a chip that can be embedded in a wheelchair. There remain, of course, several limitations, mainly concerning the geometry of the IT and soft tissues, which are not an ideal sphere and flat layer. The soft tissues are modeled as a homogenous isotropic material with no differentiation between muscle, fat, skin and connective tissues. Additionally, we assigned mechanical properties taken from animal studies of transversal loading of ovine [9] and porcine [17] fat and muscle tissues, respectively. We considered the muscle-to-fat ratio in the buttocks of each subject and weighed the elastic modulus according to the patient's soft tissue anatomy to account, in some degree, for the inter-subject variability in soft tissue stiffness. We do foresee, however, that in clinical use of the present method, it will be necessary to feed patient models with individual, directly-measured tissue mechanical properties in addition to the individual anatomy. One very promising way for obtaining the individual $E$ noninvasively and without ionizing radiation or expensive MRI is to use ultrasound elastography [6] which examines internal anatomy and tissue stiffness in a single scan. The model could be automated by developing a dedicated image processing code for segmentation of bone contours, muscle and fat layer contours, and calculating a mean radius of curvature of bone and muscle/fat ratio from the segmentation. The spatial resolution of a commercial ultrasound is in the $0.1-1 \mathrm{~mm}$ range, which should be sufficient for the present application. 
The present algorithm was tested with actual sitting pressure data recorded at the buttocks-wheelchair interface of paraplegic wheelchair users to demonstrate clinical feasibility. It should be noted that a small sample size test group was tested here. Once the system is put into large scale clinical trials it would be possible to collect population data on exposure of deep tissues to internal stresses, including time history of stresses and stress asymmetry. Through follow-up studies, this will ultimately allow to define threshold values indicating danger of DTI. In an example depicted in Fig. 5, loads measured with the pressure mat for patient D3 (Table S2) were similar under the left and right IT during the 40-65s timeframe. Contrarily, internal von Mises soft tissue stresses at that time were evaluated to be higher under the left IT. Undoubtedly, the higher tissue stresses within the left buttock in this particular subject were produced by a sharper left IT indenting a thinner soft tissue layer at that side (Table S2). In common clinical practice, the equally distributed surface pressures would have been regarded as optimal, although internal tissue stresses are not evenly distributed. This example clearly demonstrates the importance of using patientspecific models that calculate internal soft tissue stresses for DTI prevention.

The heaviest subject, W5 (Table S2), had the highest maximal and average peak internal soft tissue stresses (Table 1). This is consistent with findings of Elsner and Gefen [7] and Sopher et al. [21] who showed, using FE, that abnormal body mass indices, either overweight or underweight, contribute to the state of elevated internal tissue loads, which consequently increases the risk for sitting-acquired-DTI.

In conclusion, we propose herein a practical algorithm for real-time patientspecific monitoring of internal tissue stresses under the IT of wheelchair users, in clinical or home settings. We believe that this method holds a high potential for clinical applications, bridging between sophisticated modeling efforts at the research lab and everyday clinical needs. As a next step, we hope to translate it to a medical device ready for large-scale human studies, with the aims of screening patients at risk for DTI, based on high ISD or SAI values, and also, for training patients by means of biofeedback to avoid DTI. 


\section{Acknowledgement}

Funding for this joint Israel-France scientific collaboration was provided by the Universite Joseph Fourier (Grenoble, France) and the IDS-Arcan company (Montceau-les-Mines, France). The contribution of NV was supported by a grant from the Centre National de La Recherche Scientifique (France). 


\section{References}

1. Agam L, Gefen A (2008) Toward real-time detection of deep tissue injury risk in wheelchair users using Hertz contact theory. J Rehabil Res Dev 45:537-550.

2. Black J (2009). Deep tissue injury: an evolving science. Ostomy Wound Manage $55: 4$.

3. Bucki M, Lobos C, Payan Y (2010) A fast and robust patient specific finite element mesh registration technique: application to 60 clinical cases. Med Image Anal14:303-317.

4. Ceelen KK, Stekelenburg A, Loerakker S, Strijkers GJ, Bader DL, Nicolaij K, Baaijens FP, Oomens CW (2008) Compression-induced damage and internal tissue strains are related. J Biomech 41:3399-3404.

5. Dabnichki P, Taktak D (1998) Pressure variation under the ischial tuberosity during a push cycle. Med Eng Phys 20:242-256.

6. Deprez JF, Cloutier G, Schmitt C, Gehin C, Dittmar A, Basset O, Brusseau E (2007) 3D ultrasound elastography for early detection of lesions. Evaluation on a pressure ulcer mimicking phantom. Conference Proceedings of the IEEE Engineering in Medicine and Biology Society, pp. 79-82.

7. Elsner JJ, Gefen A (2008). Is obesity a risk factor for deep tissue injury in patients with spinal cord injury?. J Biomech 41:3322-3331.

8. Gefen A (2009) Deep tissue injury from a bioengineering point of view. Ostomy Wound Manage 55:26-36.

9. Gefen A, Haberman E (2007) Viscoelastic properties of ovine adipose tissue covering the gluteus muscles. J Biomech Eng 129:924-930.

10. Greenleaf JF, Fatemi M, Insana M (2003). Selected methods for imaging elastic properties of biological tissues. Annu Rev Biomed Eng 5:57-78.

11. Linder-Ganz E, Scheinowitz M, Yizhar Z, Margulies SS, Gefen A (2007) How do normals move during prolonged wheelchair-sitting? Technol Health Care 15:195202. 
12. Linder-Ganz E, Shabshin N, Itzchak Y, Yizhar Z, Siev-Ner I, Gefen A (2008). Strains and stresses in sub-dermal tissues of the buttocks are greater in paraplegics than in healthy during sitting. J Biomech 41:567-580.

13. Linder-Ganz E, Yarnitzky G, Yizhar Z, Siev-Ner I, Gefen A (2009) Real-time finite element monitoring of sub-dermal tissue stresses in individuals with spinal cord injury: towards prevention of pressure ulcers. Ann Biomed Eng 37:387-400.

14. Ning X, Lovell M, Slaughter WS (2006) Asymptotic solutions for axisymmetric contact of a thin, transversely isotropic elastic layer. Wear 260:693-698.

15. Olesen CG, de Zee M, Rasmussen J (2010) Missing links in pressure ulcer research - an interdisciplinary overview. J Appl Physiol 108:1458-1464.

16. Oomens CW, Loerakker S, Bader DL (2010). The importance of internal strain as opposed to interface pressure in the prevention of pressure related deep tissue injury. J Tissue Viabil 19:35-42.

17. Palevski A, Glaitch I, Portnoy S, Linder-Ganz E, Gefen A (2006) Stress relaxation of porcine gluteus muscle subjected to sudden transverse deformation as related to pressure sore modeling. J Biomech Eng 128:782-787.

18. Pipkin L, Sprigle S (2008). Effect of model design, cushion construction, and interface pressure mats on interface pressure and immersion. J Rehabil Res Dev 45:875-882.

19. Shacham S, Castel D, Gefen A (2010). Measurements of the static friction coefficient between bone and muscle tissues. J Biomech Eng 132:084502.

20. Stekelenburg A, Gawlitta D, Bader DL, Oomens CW (2008). Deep tissue injury: how deep is our understanding?. Arch Phys Med Rehab 89:1410-1413.

21. Sopher R, Nixon J, Gorecki C, Gefen A (2010) Exposure to internal muscle tissue loads under the ischial tuberosities during sitting is elevated at abnormally high or low body mass indices. J Biomech 43:280-286. 


\section{Figure captions}

\begin{tabular}{|c|c|}
\hline Fig. 1 & $\begin{array}{l}\text { (a) The geometry of the analytical model. Examples of finite element } \\
\text { simulations of a specific geometry }(R=20 \mathrm{~mm}, h=15.38 \mathrm{~mm}) \text { with } \\
\text { interface conditions of (b) free-slip and (c) no-slip. The } \alpha(R / h) \\
\text { functions for (d) free-slip and (e) no-slip simulations and the power } \\
\text { law functions fitted to each condition. }\end{array}$ \\
\hline Fig. 2 & $\begin{array}{l}\text { Synthetic simulation of static sitting where (a) the load is applied for } \\
2 \text { minutes of bilateral weight bearing, followed by load shifting to the } \\
\text { monitored side for two minutes, then } 5 \text { seconds lift-off (zero load on } \\
\text { the monitored buttock) and back to } 2 \text { minutes of bilateral weight } \\
\text { bearing. The computed von Mises stresses for variations in (b) the } \\
\text { elastic modulus and (c) the Poisson's ratio are presented as well as } \\
\text { the stress doses for variations in (d) the elastic modulus and (e) } \\
\text { Poisson's ratio. }\end{array}$ \\
\hline Fig. 3 & $\begin{array}{l}\text { Synthetic simulation of dynamic sitting during wheelchair propulsion } \\
\text { where (a) a repetitive load is applied for } 3 \text { seconds. The computed } \\
\text { von Mises stresses for variations in (b) the elastic modulus and (c) the } \\
\text { Poisson's ratio are presented as well as the stress doses for variations } \\
\text { in (d) the elastic modulus and (e) Poisson's ratio. }\end{array}$ \\
\hline Fig. 4 & $\begin{array}{l}\text { The pressure sensing area taken into account for body load } \\
\text { calculations on each side was determined by the best achieved (a) X- } \\
\text { Y plot and (b) average-difference (Bland-Altman) plot comparing } \\
\text { between von Mises stresses calculated by our algorithm against } \\
\text { patient-specific finite element von Mises stresses published by } \\
\text { Linder-Ganz et al (2008) for the same healthy }(\mathrm{n}=6 \text { ) and paraplegic } \\
(\mathrm{n}=5 \text { ) subjects during static sitting. The average-difference plot } \\
\text { shows good agreement between the two calculation methods as all } \\
\text { data points are within } \pm 2 \text { standard deviation boundaries of the } \\
\text { difference, and the absolute mean difference is } \sim 4 \mathrm{kPa} \text {. }\end{array}$ \\
\hline Fig. 5 & $\begin{array}{l}\text { An example of the (a) loads, (b) peak internal von Mises stress and } \\
\text { (c) internal stress dose under the left and right ischial tuberosities of } \\
\text { subject D3 (Table S2). }\end{array}$ \\
\hline Fig. 6 & $\begin{array}{l}\text { The stress asymmetry index (SAI) defined in eq. } 9 \text {, calculated for } 5 \\
\text { paraplegic subjects (whose characteristics are detailed in Table S2) } \\
\text { sitting in a wheelchair for } 2 \text { minutes. }\end{array}$ \\
\hline
\end{tabular}

